\title{
Effect of Tongfu Xingshen capsule on the endogenous neural stem cells of experimental rats with intracerebral hemorrhage
}

\author{
ZHIZHONG CUI ${ }^{1,2^{*}}$, SHANSHAN LIU ${ }^{3 *}$, LINGBO HOU $^{1}$, YIFAN SUN ${ }^{1}$, \\ HAOXUAN CHEN ${ }^{1}$, HUI MAO ${ }^{1}$, YUANQI ZHAO ${ }^{1,4}$ and LIJUN QIAO ${ }^{1,4}$ \\ ${ }^{1}$ Second Clinical Medical College, Guangzhou University of Chinese Medicine; ${ }^{2}$ First Clinical Medical College, \\ Guangzhou University of Chinese Medicine, Guangzhou, Guangdong 510006; ${ }^{3}$ Department of Hematology, \\ Guangzhou Institute of Pediatrics, Guangzhou Women and Children's Medical Center, Guangzhou, Guangdong 510630; \\ ${ }^{4}$ Guangdong Provincial Hospital of Chinese Medicine, Guangzhou, Guangdong 510120, P.R. China
}

Received November 11, 2020; Accepted February 18, 2021

DOI: $10.3892 / \mathrm{mmr} .2021 .12263$

\begin{abstract}
Intracerebral hemorrhage ( $\mathrm{ICH})$ can stimulate neural regeneration, promoting tissue repair and recovery of nerve function. Tongfu Xingshen capsule (TXC) is a Chinese medicinal formula used to treat $\mathrm{ICH}$ and has been shown to protect brain tissue and improve nerve function in clinical studies. However, the effect of TXC on endogenous neural stem cells (NSCs) remains elusive. To explore the mechanisms underlying TXC action, a rat model of ICH was established. The effects of TXC on the proliferation and differentiation of NSCs were assessed in the subventricular zone (SVZ). TXC significantly improved nerve function defects, decreased brain water content and restored blood-brain barrier integrity. Additionally, BrdU labeling showed that both high and low doses of TXC significantly increased the proportion of actively cycling NSCs positive for Nestin and glial fibrillary acidic protein, but did not affect the proliferation rates of NeuN-positive neurons. Finally, TXC also upregulated the mRNA levels of brain-derived neurotrophic factor and its receptor, $\operatorname{Tr} \kappa \mathrm{B}$, in affected brain tissues. Taken together, TXC accelerated neural repair and functional recovery after brain injury by potentially enhancing the proliferation and differentiation of endogenous NSCs into astroglial cells in the SVZ area.
\end{abstract}

Correspondence to: Dr Lijun Qiao or Professor Yuanqi Zhao, Guangdong Provincial Hospital of Chinese Medicine, 111 Dade Road, Guangzhou, Guangdong 510120, P.R. China

E-mail: qiaolijunwork@163.com

E-mail: tcm2008@126.com

${ }^{*}$ Contributed equally

Key words: Tongfu Xingshen capsule, hemorrhagic stroke, neural stem cells, brain protein, nutritional factor

\section{Introduction}

Intracerebral hemorrhage ( $\mathrm{ICH})$ is a type of stroke associated with higher morbidity and mortality rates (1). It is a significant cause of death and disability, with an incidence rate of 24.6 per 100,000 person-years and a fatality rate of $40 \%$ in 21 countries between 1980 and 2008 (2). ICH mainly results from the rupturing of small arterioles due to chronic hypertension (3). However, the pathophysiology of $\mathrm{ICH}$ is highly complex and involves multiple mechanisms. The poor outcome of $\mathrm{ICH}$ is attributed to both direct damages caused by hemorrhage and secondary injuries, such as brain edema, blood-brain barrier (BBB) disruption, inflammation and neuronal apoptosis (4). ICH also influences brain function, especially cognitive abilities, and may even cause cognitive decline or impairment (5). Indications for surgical treatment of ICH are restricted to a few clinically-relevant survival advantages (6). Although studies have recently focused on the pharmacological treatment of $\mathrm{ICH}$, no effective regimen has been established so far (7).

Neural stem cells (NSCs) are characterized by self-renewal and multipotent differentiation (8). They can differentiate into neurons, astrocytes and oligodendrocytes, depending on the specific stimuli received (9). NSCs located in the lateral subventricular zone (SVZ) of the adult mammalian brain generate new neurons and glia throughout life (10). Additionally, NSCs play an important role in replenishing neural cells, neurotrophy and neuro-immunoregulation (11), promoting the recovery of motion and sensory and cognitive functions following any injury (12). Interestingly, ICH stimulates NSC proliferation and differentiation in the SVZ $(13,14)$, and the nascent neurons then migrate to the damaged brain region to replace the dead neurons. Since neurogenesis plays a pivotal role in facilitating neurological recovery after stroke (15), NSC-based therapy has gained considerable attention for treating hemorrhagic stroke. A study demonstrated that NSC transplantation could promote the functional recovery of ICH rats (16). It has also been reported that endogenous NSCs are activated in the brain of experimental $\mathrm{ICH}$ rats and help neurons achieve 
self-repair (17). Neurotrophic factors, such as brain-derived neurotrophic factor (BDNF) and its receptor $(\operatorname{Tr} \kappa B)$, promote the proliferation and differentiation of endogenous NSCs (18) and may augment lesion repair.

Traditional Chinese medicine (TCM) has been used to improve health, prevent diseases, and treat serious illnesses for thousands of years in China and other Asian countries $(19,20)$. The formulations consist of multiple herbs that target several disease components by following the TCM theory (21). Tongfu Xingshen capsules (TXC) are composed of senna leaf, giant knotweed rhizome, tabasheer, snake gourd seed and artificial bezoar to purge fu-organs to arouse the spirit, clear heat and resolve phlegm. It was first prescribed by Professor Liu of Guangdong Hospital of Traditional Chinese Medicine for treating hemorrhagic stroke as part of the 'Tongfu-Xiedu-Xingnao-Kaiqiao' treatment model. TXC can promote recovery after cerebral hemorrhage, improve capillary permeability of the hemorrhagic area, and alleviate cerebral edema. The absorption of hematoma can relieve pressure on the brain tissue, improve cerebral microcirculation and oxygen supply, and eventually restore neurological functions (22-25). In the present study, the mechanisms underlying the therapeutic effect of TXC was assessed in a rat model of $\mathrm{ICH}$, with a specific focus on the neurological function score and the proliferation and differentiation of endogenous NSCs.

\section{Materials and methods}

Chemicals and reagents. Tongfu Xingshen capsules (TXC; $0.4 \mathrm{~g} / \mathrm{granule}$ ) were provided by the Pharmacy Department of Guangdong Hospital of Traditional Chinese Medicine. Evans Blue (EB), BrdU, formamide and sodium pentobarbital were purchased from Guangzhou Weijia Technology Co. Ltd. All chemicals and solvents were of an analytical grade.

Establishment of an ICH model and drug administration. Male SPF grade SD rats (age, 6 weeks) weighing between 200 and $250 \mathrm{~g}$ were purchased from the Institute of Experimental Animals, Chinese Academy of Medical Sciences (Beijing, China), and acclimatized in standard cages under controlled temperature $\left(23 \pm 3^{\circ} \mathrm{C}\right)$ and a regular $12 \mathrm{~h}: 12 \mathrm{~h}$ light-dark cycle for 7 days before the procedure. All experimental animal protocols were approved and performed following the Institutional Animal Care Guidelines (approval no. 2016040; Experimental Animal Ethics Committee, Guangdong Provincial Hospital of Chinese Medicine, Guangzhou, China).

The animals were divided into the sham-operated, untreated model, low dose TXC-treated, and high dose TXC-treated groups, and ICH was induced through autologous intracerebral blood infusion. In brief, the animals were all anesthetized using pentobarbital $(50 \mathrm{mg} / \mathrm{kg}$ i.p.), and $50 \mu \mathrm{l}$ blood was drawn from the tail artery. The rats were positioned in a stereotaxic frame (Stoelting Instruments), and a cranial burr hole $(1 \mathrm{~mm})$ was drilled near the right coronal suture $3.0 \mathrm{~mm}$ lateral to the midline. A microsyringe was stereotaxically inserted into the right basal ganglia (coordinates: $0.2 \mathrm{~mm}$ anterior, $6.0 \mathrm{~mm}$ ventral, and $3.0 \mathrm{~mm}$ lateral to the bregma), and autologous whole blood was injected at the rate of $10 \mu \mathrm{l} / \mathrm{min}$ (26-28). In the sham-operated control, the blood was also drawn from the tail artery before the needle was inserted without injecting any blood. The rats were intragastrically administered $1 \mathrm{ml} / 100 \mathrm{~g}$ normal saline (sham-operated

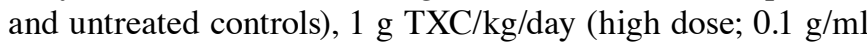
with normal saline), or $0.5 \mathrm{~g} \mathrm{TXC} / \mathrm{kg} / \mathrm{day}$ (low dose; $0.05 \mathrm{~g} / \mathrm{ml}$ with normal saline), as appropriate. For testing neurological function score, brain water content and vascular permeability test, $96 \mathrm{SD}$ rats were divided as aforementioned $(n=24$ per group). Each group was further subdivided into the 1, 7, 14 and 28-day groups ( $\mathrm{n}=6$ per group). For immunofluorescence and reverse transcription-quantitative PCR (RT-qPCR), 72 animals were similarly divided into 7, 14 and 28-day subgroups in each treatment group.

BrdU labeling. $\mathrm{BrdU}(50 \mathrm{mg} / \mathrm{kg})$ was intraperitoneally injected at a concentration of $5 \mathrm{mg} / \mathrm{ml}$ in $0.9 \% \mathrm{NaCl}$, once a day. Except for the 1-day group, the other groups received daily injections for 5 days after the model was established.

Tissue collection. From each rat, $6-\mu \mathrm{m}$ thick serial coronal sections of the brain were cut to include the entire SVZ (80-90 slides per animal, and 3 sections per slide). Every tenth slide was stained using hematoxylin and eosin at room temperature following standard protocols. Briefly, brain sections were stained with hematoxylin for 5 min and differentiated with $1 \%$ hydrochloric acid alcohol for $5 \mathrm{sec}$ at room temperature. Following immersion in ammonia-water for $10 \mathrm{sec}$, sections were dyed with eosin for $3 \mathrm{~min}$, followed by mounting with neutral balsam. After sealing, the SVZ structures were observed at X40 magnification under an Olympus BX61 light microscope, and adjacent sections with similar SVZ structures were used for double immunofluorescence staining.

Behavioral testing. Neurological tests were performed $24 \mathrm{~h}$ post-stroke and were scored according to the modified Bederson scale as follows: 0 , no deficits; 1 , flexed forepaw; 2 , an inability to resist the lateral push; 3 , circling; 4, agitated circling; and 5, unresponsive to stimulation/stupor (29). The neurobehavioral score of 1-5 was considered as a successful model.

Evan's Blue dye extravasation assay. Evan's Blue dye extravasation test was performed, as previously described (30). The harvested brains were quickly separated into the left anterior, right anterior, left posterior and right posterior segments, weighed and digested using $3 \mathrm{ml}$ formamide in a $60^{\circ} \mathrm{C}$ water bath for $24 \mathrm{~h}$. The homogenates were centrifuged at $10,000 \mathrm{x} g$ at room temperature for $20 \mathrm{~min}$, and the absorbance of the supernatant at $630 \mathrm{~nm}$ was measured using a spectrophotometer. The Evans blue content was calculated as $\mathrm{mg} / \mathrm{g}$ against a standard curve.

Brain water content. Rats were decapitated under deep anesthesia (pentobarbital; $50 \mathrm{mg} / \mathrm{kg}$ i.p.), which was validated based on the fact that there was no pain reaction, no stimulation reaction, general muscle relaxation, and smooth breathing, and the brains were immediately removed. The posterior brains were weighed using an analytical microbalance to obtain the wet weight (WW). The samples were then dried at $75^{\circ} \mathrm{C}$ for 5 days, and the dry weight (DW) was determined. Brain water content was calculated as (WW-DW)/WW x100\%. 
Double immunofluorescence. NeuN/BrdU and GFAP/BrdU double staining were performed on the 14-day and 28-day samples, while the 7-day samples were stained using Nestin and BrdU. In brief, the sections were incubated overnight with rat anti-BrdU (cat. no. ab6326) and mouse anti-Nestin (cat. no. ab6142), rabbit anti-NeuN (cat. no. ab177487) or rabbit anti-GFAP (all 1:200; cat. no. ab33922; all Abcam), as appropriate, at $4^{\circ} \mathrm{C}$. After probing with the relevant secondary antibody (all 1:500; Alexa Fluor ${ }^{\circledR} 488$ donkey anti-rat, cat. no. A21208; anti-mouse, cat. no. A32744 or anti-rabbit IgG, cat. no. A32754; Thermo Fisher Scientific, Inc.) at room temperature for $1 \mathrm{~h}$ in the dark, the slides were washed and observed at x400 magnification under a Nikon Ti2-E light microscope. ImageJ software (v2.1.4.7; National Institutes of Health) was used to measure the number of positive cells. The number of cells in the SVZ region of 3 non-overlapping fields were counted in each section, and the mean was calculated.

$R N A$ preparation and $R T-q P C R$ analysis. Total RNA was extracted from the brain tissues using the TRIzol reagent, following the manufacturer's instructions (Takara Biotechnology Co., Ltd.). The following primers were used to perform the RT-qPCR: Rat-BDNF forward primer, GATTAGGTGGCT TCATAGGAGAC; rat-BDNF reverse primer, CGAACAGAA ACAGAGGAGAGATT; rat-TrkB forward primer, GATGTT CCAGCCACTGTGAACC; rat-TrkB reverse primer, TCC ACCACCCTGTTGCTGTA. Rat-GADPH (forward, 5'-GAT GTTCCAGCCACTGTGAACC-3' and reverse, 5'-TCCACC ACCCTGTTGCTGTA-3') was used as the internal control. The purity of RNA samples was assessed via NanoDrop, and samples with ratios between 1.80 and 2.01 were used for cDNA synthesis using the GoScript ${ }^{\mathrm{TM}}$ Reverse Transcription system, according to the manufacturer's protocol (Promega Corporation). The qPCR reaction was performed in $20 \mu \mathrm{l}$ with $5 \mu \mathrm{l}$ template DNA and $500 \mathrm{nM}$ primers. The PCR condition was as follows: Initial denaturation, $95^{\circ} \mathrm{C}$ for $10 \mathrm{~min}, 40$ cycles of amplification $\left(95^{\circ} \mathrm{C}\right.$ for $10 \mathrm{sec}$ and $60^{\circ} \mathrm{C}$ for $\left.30 \mathrm{sec}\right)$ and cooling period, $50^{\circ} \mathrm{C}$ for $5 \mathrm{sec}$. Each sample was analyzed in triplicate. iTaq ${ }^{\mathrm{TM}}$ Universal SYBR ${ }^{\circledR}$ Green Supermix (Bio-Rad Laboratories, Inc.) was used according to the manufacturer's instruction. The PCR results were analyzed in a Real-Time PCR system (Bio-Rad Laboratories, Inc.; cat. no. CFX96). The specificity of the primers was tested by analyzing the dissolution curve. The mRNA of each target gene was homogenized, and the phase of mRNA of each target gene was calculated by $\mathrm{Cq}$ value for the relative expression level $\left(2^{-\Delta \Delta C q}\right.$ method: $\Delta \Delta \mathrm{Cq}=\left(\mathrm{Cq}_{\text {target gene }}-\mathrm{Cq}\right.$ GAPDH $\left.)_{\text {treatment group }}-\left(\mathrm{Cq}_{\text {target gene }}-\mathrm{Cq}_{\mathrm{GAPDH}}\right)_{\text {control group }}\right)(31)$.

Statistical analysis. The data were analyzed using SPSS 21 software (IBM Corp.) and are expressed as the mean \pm SD Differences were analyzed using one-way analysis of variance ANOVA with post hoc Bonferroni test. $\mathrm{P}<0.05$ was considered to indicate a statistically significant difference.

\section{Results}

TXC alleviates neurological deficit and structural damage following ICH. ICH resulted in left hemiplegia, severe hemiplegia in the left forelimb and clockwise rear-ended rotation within 7 days of induction, and the symptoms were observed
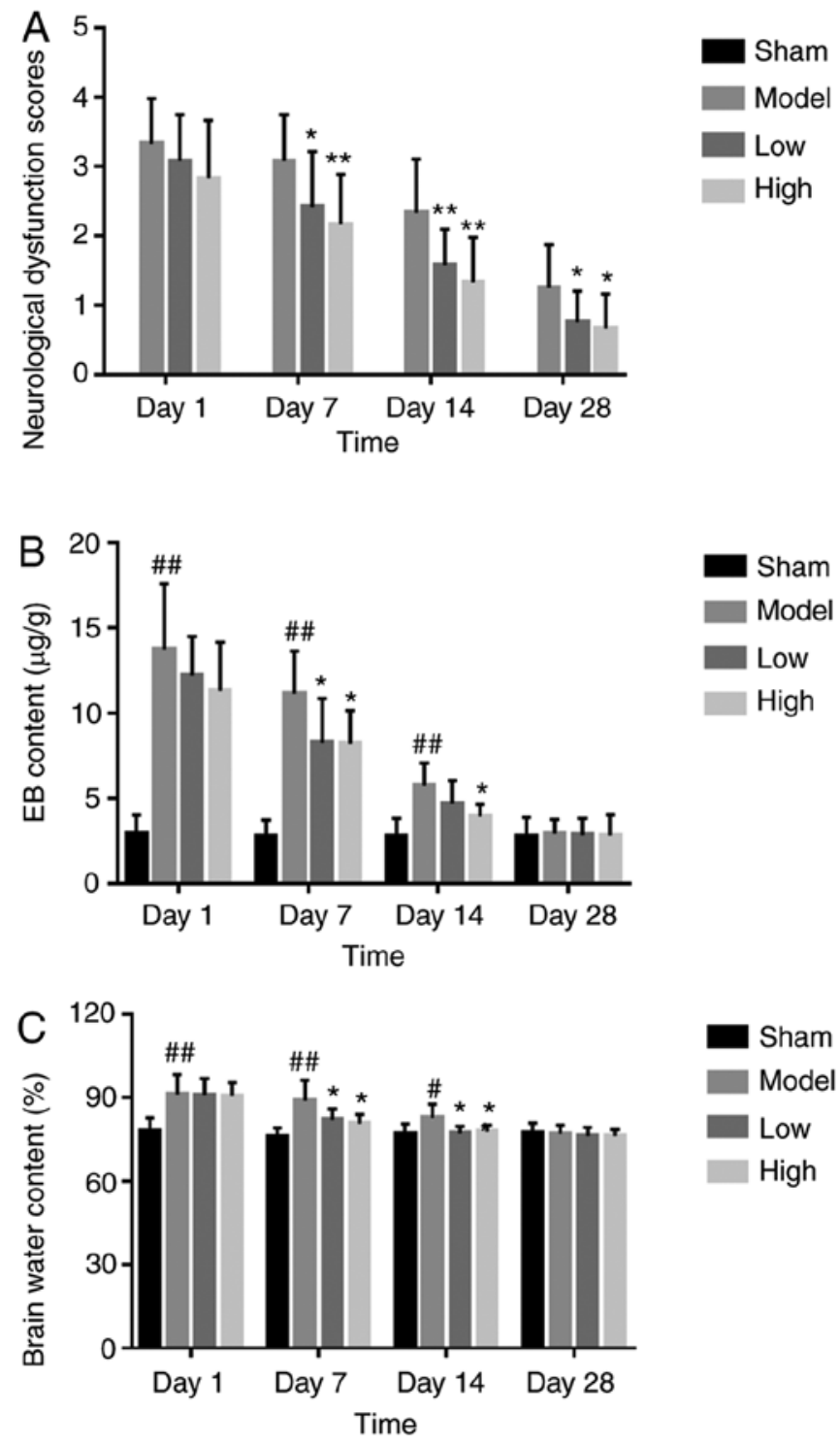

Figure 1. (A) Neurological scores in the groups indicated. (B) Evans Blue content of hemorrhagic brain tissue in the different groups. (C) Brain water content in the different groups. ${ }^{\#} \mathrm{P}<0.05$ and ${ }^{\# \prime} \mathrm{P}<0.01$ vs. sham group; ${ }^{*} \mathrm{P}<0.05$ and ${ }^{* *} \mathrm{P}<0.01$ vs. model group.

over 14 and 28 days. Compared with the untreated model group, both low and high dosages of TXC improved gait symptoms and decreased hemiplegia (Fig. 1A). Furthermore, TCX significantly decreased the extravasation of EB from brain tissues after 7, 14 and 28 days of stroke, compared with the untreated model group (Fig. 1B). Finally, the brain water content in the ipsilateral hemicerebrum substantially increased in the model group, compared with the sham-operated animals, and was significantly decreased by low and high dose TXC treatment at all time points (Fig. 1C).

TXC promotes the proliferation and differentiation of endogenous NSCs in SVZ. ICH injury significantly increased the number of proliferating BrdU-positive cells in the SVZ region, increased by low or high dose TXC-treated (Fig. 2). As shown in Fig. 3, the total number of proliferating NSCs (Nestin- and BrdU-positive cells) was markedly increased after TXC treatment within 7 days post-stroke. However, the proportion of actively cycling neuron (NeuN- and BrdU-positive) 

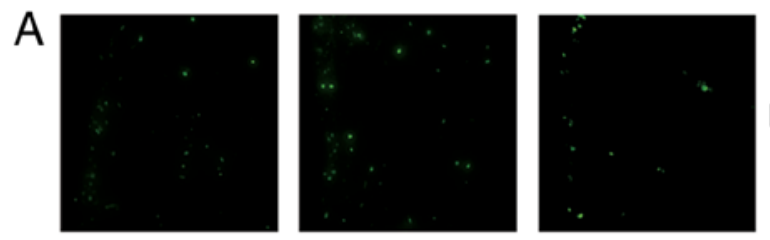

High
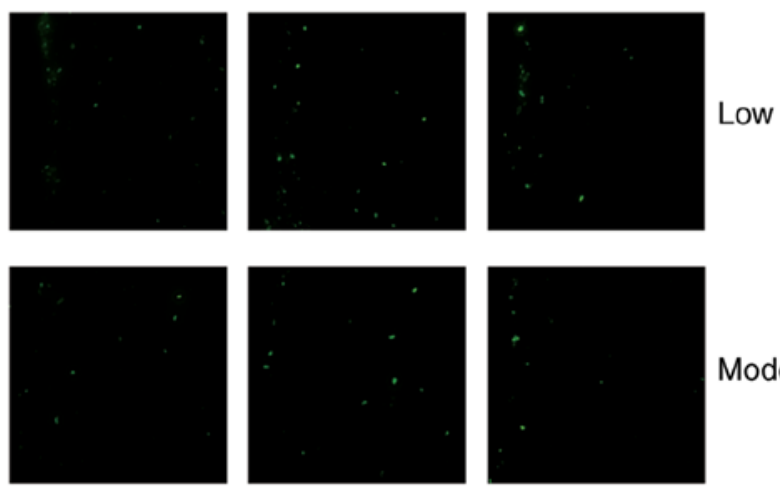

Model

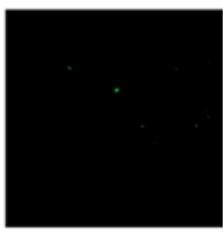

7

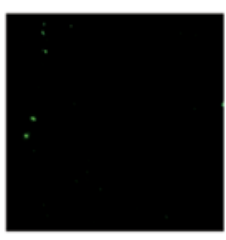

14

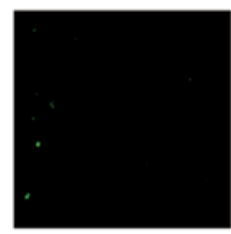

28

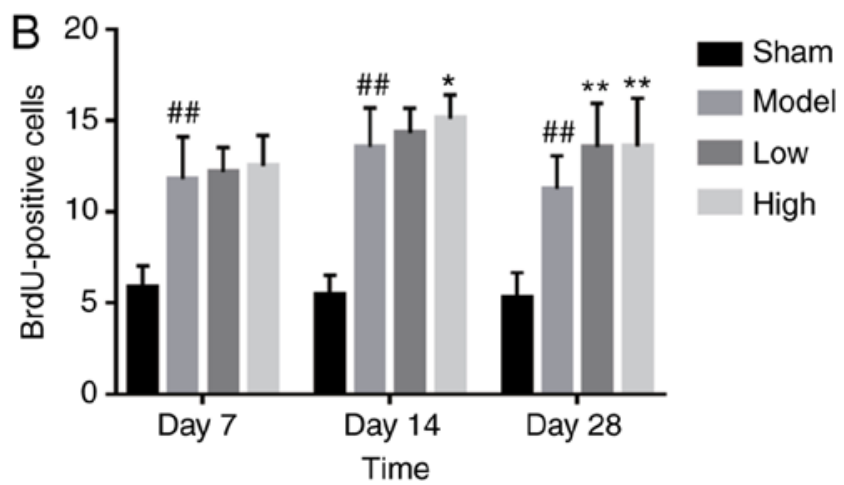

Figure 2. (A) Representative immunofluorescence images showing BrdU-positive cells (magnification, $x 400 ; n=3$ ). (B) The number of BrdU-positive cells in the SVZ of the different groups. ${ }^{\# \#} \mathrm{P}<0.01$ vs. sham group; ${ }^{*} \mathrm{P}<0.05$ and ${ }^{* *} \mathrm{P}<0.01$ vs. model group.

cells was similar in both the untreated and treated groups, both at 14 and 28 days post-stroke (Fig. 4). Furthermore, TXC treatment also increased the number of proliferating glial cells (GFAP- and BrdU-positive) in the SVZ region after 14 and 28 days post-stroke (Fig. 5 ).

TXC upregulates BDNF and TrkB after ICH. The BDNF mRNA levels were significantly higher in the brain tissues of the TXC-treated animals compared with the untreated controls at 7 and 14 days post-stroke. The upregulation on day 14 was particularly notable in the high dose TXC group. However, there was no significant difference in BDNF expression between the untreated and treated groups after 28 days (Fig. 6A). TrkB mRNA was also upregulated in the model group compared with the sham-operated group after 7 days $(\mathrm{P}<0.05)$, and this effect was augmented further by high-dose TXC treatment on day 7 and day 28 (Fig. 6B).
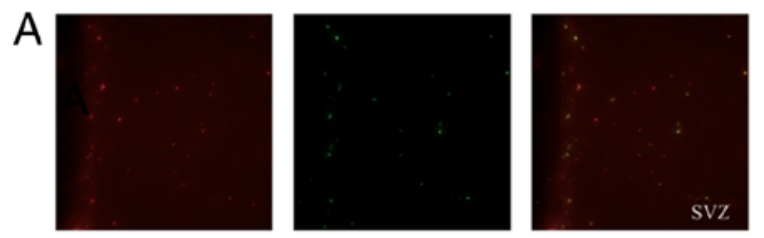

High
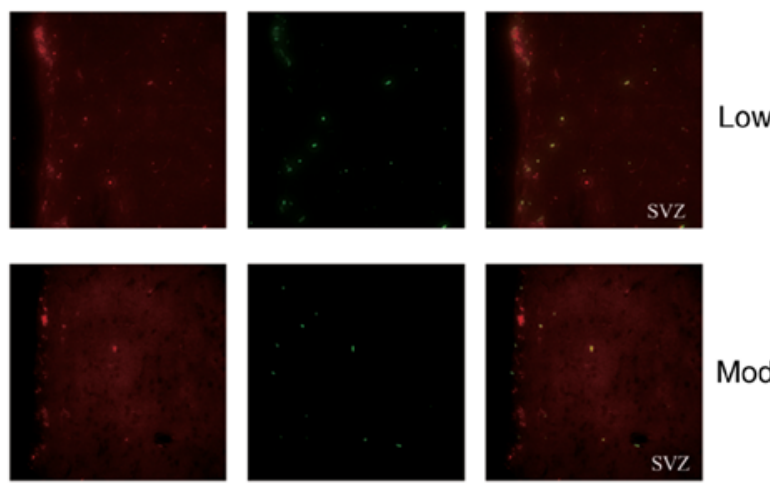

Model

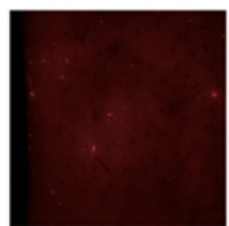

Nestin

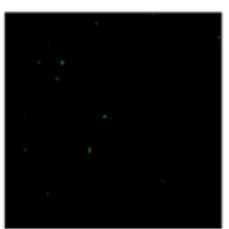

BrdU

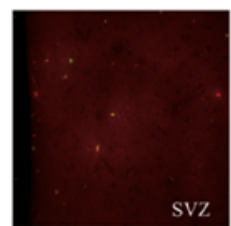

Nestin/BrdU
Sham

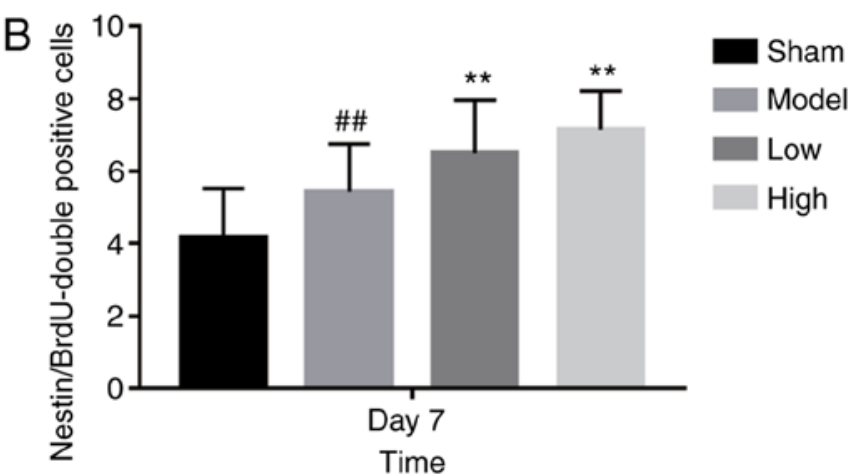

Figure 3. (A) Representative immunofluorescence images showing Nestinand BrdU-positive cells (magnification, $x 400 ; n=3$ ). (B) The number of Nestin- and BrdU-positive cells in the subventricular zone of the different groups. ${ }^{\# \#} \mathrm{P}<0.01$ vs. sham group; ${ }^{* *} \mathrm{P}<0.01$ vs. model group.

\section{Discussion}

It was found that EB extravasation in the brain was most significant on the first-day post-stroke and decreased along with time. Brain edema following ICH is mainly attributed to the disruption of the BBB (32). However, currently, drugs that can significantly improve BBB function after stroke have not yet been identified. TXC significantly inhibited EB extravasation, indicating that it can improve cerebral vascular permeability and alleviate brain edema of the affected rats in a dose-dependent manner during ICH.

A few BrdU-positive cells were detected in the ependymal epithelium of the SVZ region at different time points in the sham-operated group, which was consistent with the small number of resting endogenous NSCs previously reported in this region (13). In the present study, ICH significantly 
A
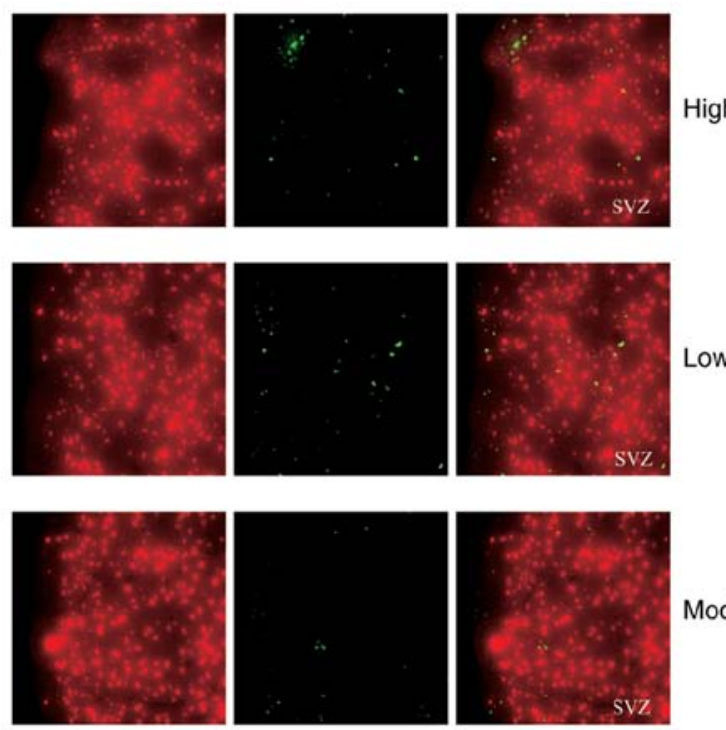

Model

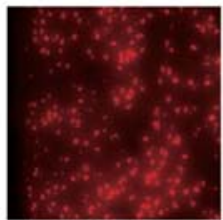

NeuN

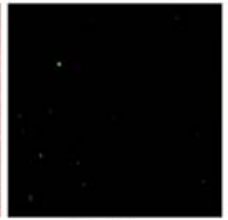

BrdU

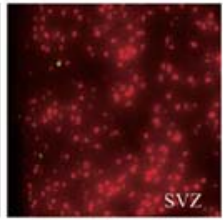

NeuN/BrdU

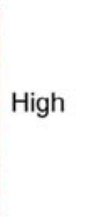

Low
B
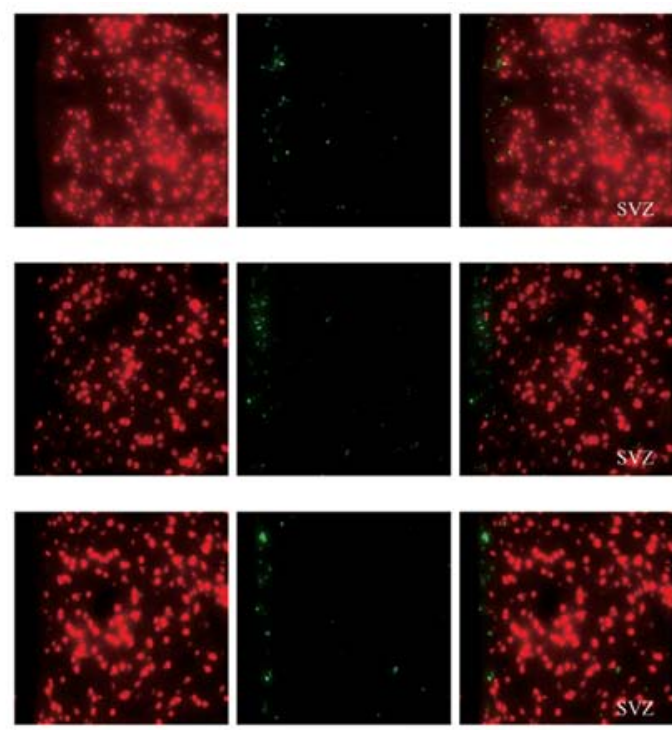

Sham

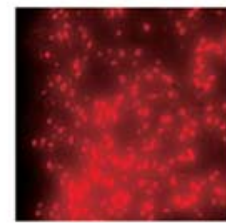

NeuN

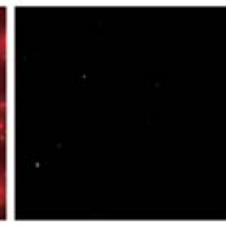

BrdU

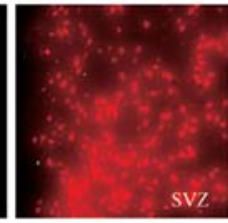

NeuN/BrdU

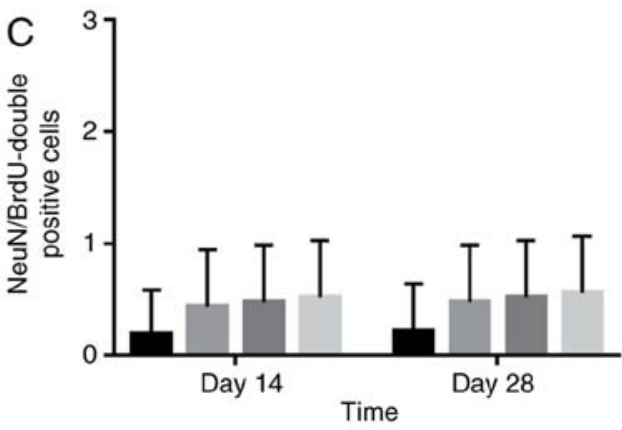

Figure 4. Representative immunofluorescence images showing NeuN- and BrdU-positive cells on (A) day 14 and (B) day 28 (magnification, $\mathrm{x} 400$; $\mathrm{n}=3$ ). (C) The number of NeuN- and BrdU-positive cells in the subventricular zone of the different groups.

increased the number of proliferating Nestin-positive NSCs on the 7th day, which further enhanced the TXC treatment effect. Furthermore, significant glial cell proliferation was observed in the TXC-treated animals on the 14 and 28th days after TXC administration. TXC promoted NSC proliferation and differentiation into astroglial cells but not into neurons. However, the proliferation and activation of astrocytes were also beneficial for ICH. Astrocytes act as a 'double-edged sword' inactivating NSCs after stroke. During the early stage of stroke, astrocytes can restrict the diffusion of inflammatory factors by secreting various neurotrophic factors that protect nascent neurons. During the later stages, excessive proliferation and activation of astrocytes can mechanically hinder neural regeneration by forming an extensive network (33).

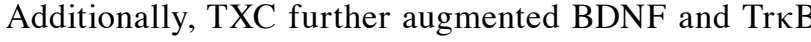
levels, which likely increased NSC proliferation and differentiation in the SVZ, although the exact mechanism is still unclear. Studies have shown that BDNF and its receptor, $\operatorname{Tr} \kappa \mathrm{B}$, are upregulated following cerebral ischemia and epilepsy $(34,35)$. Brain transplantation of human NSCs overexpressing BDNF provided differentiation and survival abilities to the grafted human NSCs and renewed angiogenesis in the host brain and functional recovery of ICH animals (36). The simultaneous increase in astrocyte proliferation and $\mathrm{BDNF} / \mathrm{Tr} \kappa \mathrm{B}$ expression after ICH suggests that activated glial cells secrete neurotrophic factors, which may drive pathological changes associated with $\mathrm{ICH}$ and promote neuronal survival. However, BDNF protein is mainly expressed in activated microglia around hematoma. In contrast, BDNF mRNA is mainly expressed in neurons and partially expressed in activated microglia around hematoma (37), which may be closely associated with the secretion of various neurotrophic factors by activated glial cells (38).

Taken together, the present findings demonstrated that TXC could significantly improve neurological deficits, absorption of brain edema, BBB integrity and NSC proliferation and differentiation into glial cells by upregulating the neuroprotective factors, BDNF and TrkB. This study showed that TXC might 

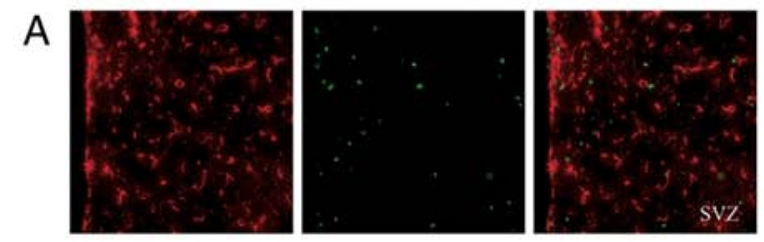

High

B
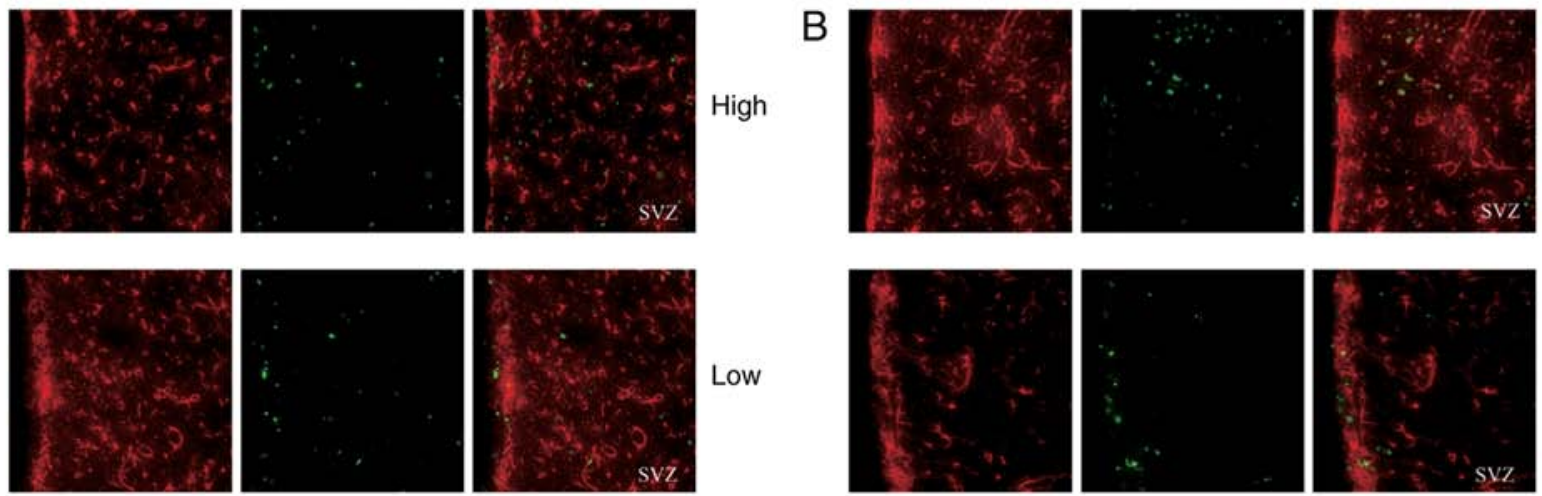

Low
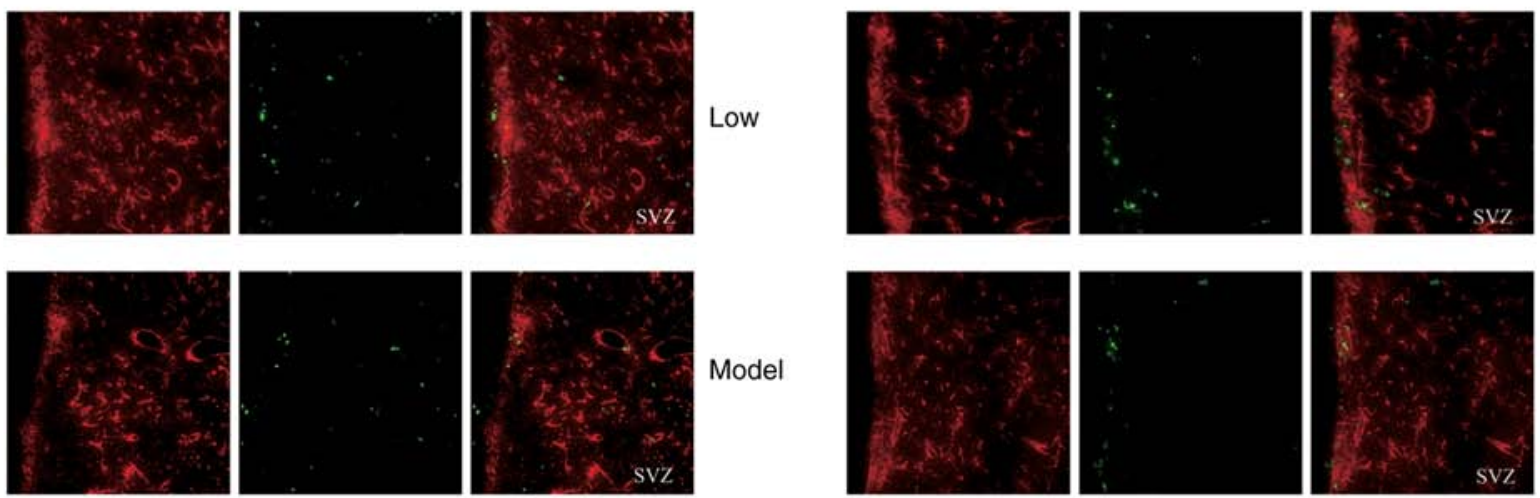

Model
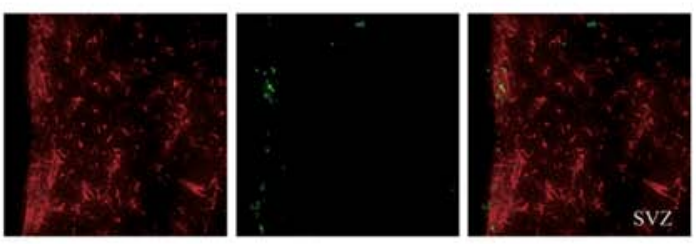

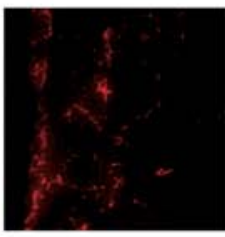

GFAP

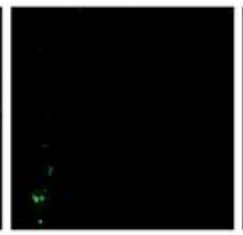

BrdU

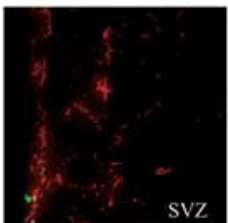

GFAP/BrdU
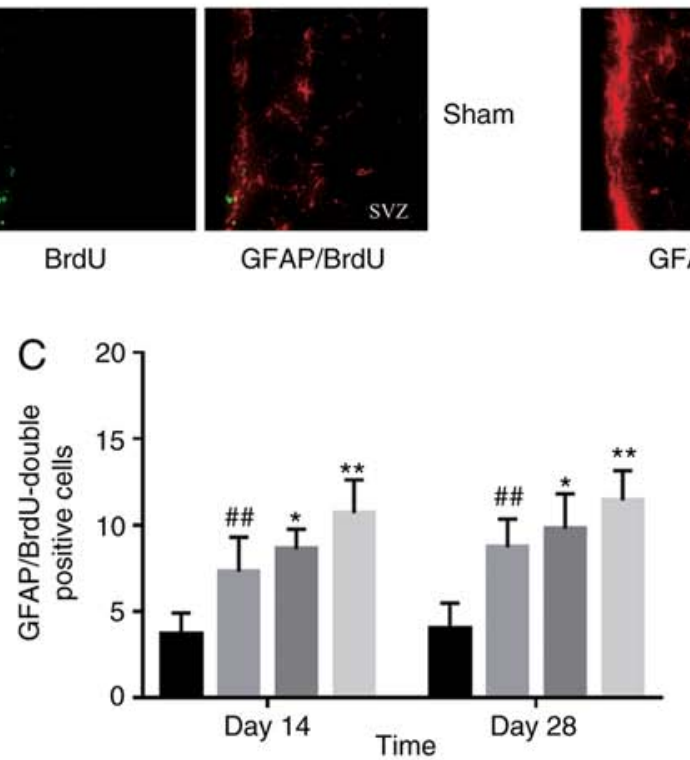

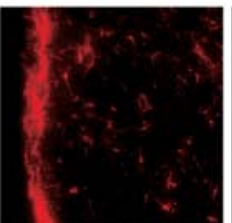

GFAP

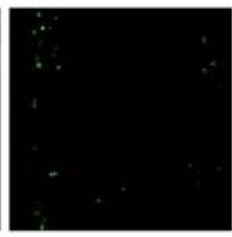

BrdU

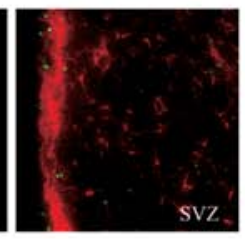

GFAP/BrdU

Figure 5. (A) Representative immunofluorescence images showing GFAP- and BrdU-positive cells on day 14 and (B) day 28 (magnification, $x 400 ; n=3$ ). (C) The number of GFAP- and BrdU-positive cells in the subventricular zone of the different groups. ${ }^{\# \#} \mathrm{P}<0.01$ vs. sham group; ${ }^{*} \mathrm{P}<0.05$ and ${ }^{* * *} \mathrm{P}<0.01$ vs. model group. GFAP, glial fibrillary acidic protein.
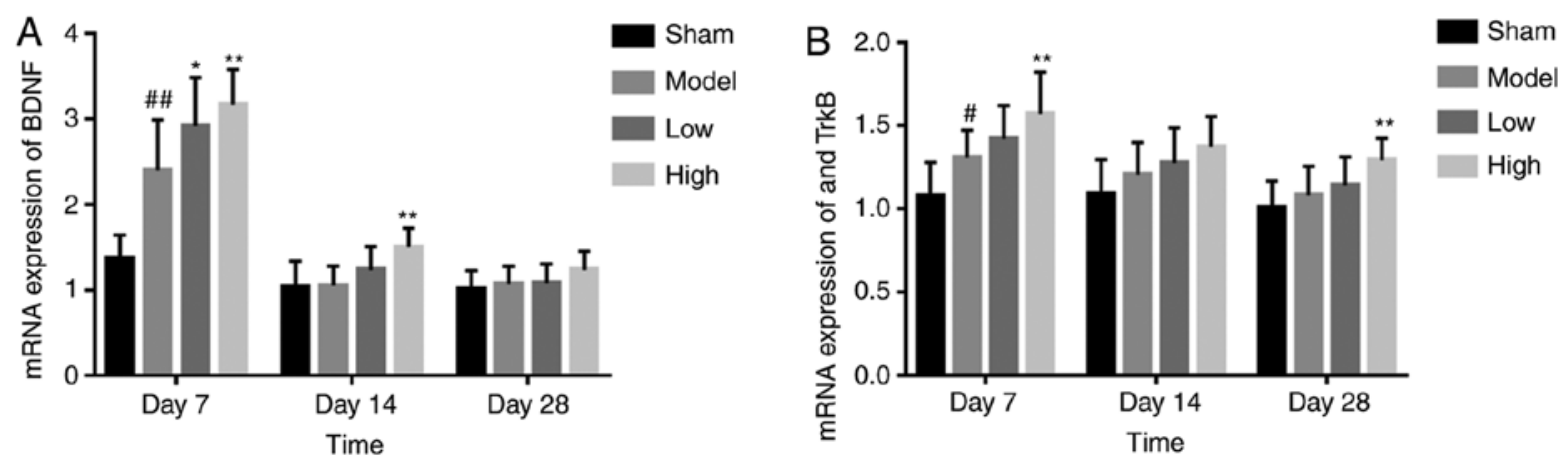

Figure 6. (A) BDNF mRNA expression in the SVZ of the different groups. (B) TrkB mRNA expression in the $\mathrm{SVZ}$ of the different groups. ${ }^{\#} \mathrm{P}<0.05$ and ${ }^{\# \#} \mathrm{P}<0.01$ vs. sham group; ${ }^{\mathrm{P}}<0.05$ and ${ }^{* *} \mathrm{P}<0.01$ vs. model group. $\mathrm{SVZ}$, subventricular zone. BDNF, brain-derived neurotrophic factor.

be an effective treatment method using an ICH rat model and provided a novel perspective on the treatment and prevention of ICH in patients. However, further clinical studies will be necessary to confirm these results. 


\section{Acknowledgements}

The authors would like to thank Professor Xiao Cheng (Second Clinical Medical College; Guangzhou University of Chinese Medicine, Guangzhou, China) for editing this manuscript, critical reading and providing suggestions.

\section{Funding}

The present study was supported by the Guangdong Natural Science Foundation (grant nos. 2015A030310436 and 2018A0303130053 to LQ).

\section{Availability of data and materials}

The datasets used and/or analyzed during the current study are available from the corresponding author on reasonable request.

\section{Authors' contributions}

LQ and YZ conceived and designed the experiments. ZC and SL performed the experiments. LH, YS, HC and HM analyzed the data. The manuscript was drafted by $\mathrm{ZC}$ and $\mathrm{SL}$ and revised by all authors. ZC and SL confirm the authenticity of all the raw data. All authors have read and approved the manuscript and have contributed significantly to the study.

\section{Ethics approval and consent to participate}

All experimental animal protocols were approved and performed following the Institutional Animal Care Guidelines (approval nr. 2016040; Experimental Animal Ethics Committee, Guangdong Provincial Hospital of Chinese Medicine, Guangzhou, China).

\section{Patient consent for publication}

Not applicable.

\section{Competing interests}

The authors declare that they have no competing interests.

\section{References}

1. Huang AP, Hsu YH, Wu MS, Tsai HH, Su CY, Ling TY, Hsu SH and Lai DM: Potential of stem cell therapy in intracerebral hemorrhage. Mol Biol Rep 47: 4671-4680, 2020.

2. Gregorio T, Pipa S, Cavaleiro P, Atanásio G, Albuquerque I, Chaves PC and Azevedo L: Prognostic models for intracerebral hemorrhage: Systematic review and meta-analysis. Bmc Med Res Methodol 18: 145, 2018

3. An SJ, Kim TJ and Yoon BW: Epidemiology, risk factors, and clinical features of intracerebral hemorrhage: An update. J Stroke 19: 3-10, 2017.

4. Wu TY, Sharma G, Strbian D, Putaala J, Desmond PM, Tatlisumak T, Davis SM and Meretoja A: Natural history of perihematomal edema and impact on outcome after intracerebral hemorrhage. Stroke 48: 873-879, 2017.

5. Garcia PY, Roussel M, Bugnicourt JM, Lamy C, Canaple S, Peltier J, Loas G, Deramond H and Godefroy O: Cognitive impairment and dementia after intracerebral hemorrhage: A cross-sectional study of a hospital-based series. J Stroke Cerebrovasc Dis 22: 80-86, 2013.
6. Pias-Peleteiro J, Campos F, Castillo J and Sobrino T: Endothelial progenitor cells as a therapeutic option in intracerebral hemorrhage. Neural Regen Res 12: 558-561, 2017.

7. Wang Z, Zhou F, Dou Y, Tian X, Liu C, Li H, Shen H and Chen G: Melatonin alleviates intracerebral hemorrhage-induced secondary brain injury in rats via suppressing apoptosis, inflammation, oxidative stress, DNA damage, and mitochondria injury. Transl Stroke Res 9: 74-91, 2018.

8. Hu YD, Zhao Q, Zhang XR, Xiong LL, Zhang ZB, Zhang P, Zhang RP and Wang TH: Comparison of the properties of neural stem cells of the hippocampus in the tree shrew and rat in vitro. Mol Med Rep 17: 5676-5683, 2018.

9. Suksuphew S and Noisa P: Neural stem cells could serve as a therapeutic material for age-related neurodegenerative diseases. World J Stem Cells 7: 502-511, 2015.

10. Fuentealba LC, Obernier K and Alvarez-Buylla A: Adult neural stem cells bridge their niche. Cell Stem Cell 10: 698-708, 2012.

11. Dooley D, Vidal P and Hendrix S: Immunopharmacological intervention for successful neural stem cell therapy: New perspectives in CNS neurogenesis and repair. Pharmacol Ther 141: 21-31, 2014.

12. Stenudd M, Sabelstrom H and Frisen J: Role of endogenous neural stem cells in spinal cord injury and repair. JAMA Neurol 72: 235-237, 2015.

13. Shen J, Xie L, Mao X, Zhou Y, Zhan R, Greenberg DA and Jin K: Neurogenesis after primary intracerebral hemorrhage in adult human brain. J Cereb Blood Flow Metab 28: 1460-1468, 2008.

14. Masuda T, Isobe Y, Aihara N, Furuyama F, Misumi S, Kim TS, Nishino $\mathrm{H}$ and Hida $\mathrm{H}$ : Increase in neurogenesis and neuroblast migration after a small intracerebral hemorrhage in rats. Neurosci Lett 425: 114-119, 2007.

15. Yang P, Cai L, Zhang G, Bian Z and Han G: The role of the miR-17-92 cluster in neurogenesis and angiogenesis in the central nervous system of adults. J Neurosci Res 95: 1574-1581, 2017.

16. Wakai T, Narasimhan P, Sakata H, Wang E, Yoshioka $H$, Kinouchi $\mathrm{H}$ and Chan $\mathrm{PH}$ : Hypoxic preconditioning enhances neural stem cell transplantation therapy after intracerebral hemorrhage in mice. J Cereb Blood Flow Metab 36: 2134-2145, 2016.

17. Cui M, Ge H, Zeng H, Yan H, Zhang L, Feng H and Chen Y: Repetitive transcranial magnetic stimulation promotes neural stem cell proliferation and differentiation after intracerebral hemorrhage in mice. Cell Transplant 28: 568-584, 2019.

18. Schabitz WR, Berger C, Kollmar R, Seitz M, Tanay E, Kiessling M, Schwab S and Sommer C: Effect of brain-derived neurotrophic factor treatment and forced arm use on functional motor recovery after small cortical ischemia. Stroke 35: 992-997, 2004.

19. Bochorakova H, Paulova H, Slanina J, Musil P and Taborska E: Main flavonoids in the root of scutellaria baicalensis cultivated in Europe and their comparative antiradical properties. Phytother Res 17: 640-644, 2003.

20. Wang CZ, Mehendale SR, Calway T and Yuan CS: Botanical flavonoids on coronary heart disease. Am J Chin Med 39: 661-671, 2011.

21. Fu X, Lu R and Zhao S: Simultaneous quantitation of six aconitum alkaloids and three flavonoids in the herb couple of radix aconiti lateralis-radix glycyrrhizae (Fuzi-Gancao) by UHPLC-ESI-MS/MS. Pharmacogn Mag 13: 425-429, 2017.

22. Wang L, Liu M, Lu B and Sun J: Effect of tongfu xingshen liquid on the expression of HO-1 mRNA and HSP70 in cerebral tissue of rats with intracerebral hemorrhage. J Chengdu Unversity of Tarditional Chin Med 2: 27-29, 2004 (In Chinese).

23. Hui QX, Zhi ZR and Hua L: Study on qualitative and quantitative methods of tongfu xingshen capsule. Chin J Exp Traditional Med Formulae 7: 22-24, 2001 (In Chinese).

24. Cai LM, Xun LB and Bo SJ: Effect of tongfu xingshen liquid enema on cerebral edema and cerebral vascular permeability in rats with intracerebral hemorrhage. Chin J Information on TCM 11: 210-212, 2004 (In Chinese).

25. Jingbo S, Rong H, Peixin $\mathrm{H}$ and Yan $\mathrm{H}$ : Effects of tongfu xingshen capsule on the animal model of stroke with tanre fushi syndrome in rats. Chin J Integrated Traditional Chin Western Medicine First Aid 06: 341-343, 2001 (In Chinese).

26. Nath FP, Jenkins A, Mendelow AD, Graham DI and Teasdale GM: Early hemodynamic changes in experimental intracerebral hemorrhage. J Neurosurg 65: 697-703, 1986.

27. Yu YY, Niu L, Gao L, Zhang GL, Li J, Deng JP, Qu YZ, Zhao ZW and Gao GD: Ferrous chelator 2,2'-dipyridyl attenuates cerebral vasospasm after experimental subarachnoid haemorrhage in rabbits. J Int Med Res 38: 583-592, 2010. 
28. Manaenko A, Chen H, Zhang JH and Tang J: Comparison of different preclinical models of intracerebral hemorrhage. Acta Neurochir Suppl 111: 9-14, 2011.

29. Bederson JB, Pitts LH, Tsuji M, Nishimura MC, Davis RL and Bartkowski H: Rat middle cerebral artery occlusion: Evaluation of the model and development of a neurologic examination. Stroke 17: 472-476, 1986.

30. Bechet S, Hill F, Gilheaney O and Walshe M: Diagnostic accuracy of the modified evan's blue dye test in detecting aspiration in patients with tracheostomy: A systematic review of the evidence. Dysphagia 31: 721-729, 2016.

31. Livak KJ and Schmittgen TD: Analysis of relative gene expression data using real-time quantitative PCR and the 2(-Delta Delta C(T)) method. Methods 25: 402-408, 2001.

32. Jin JH, Jie WJ, Qiang X, Fen ZJ and Yu XX: Influence factors and mechanism of Borneol on blood brain barrier permeability. Chin J Chin Materia Medica 42: 2200-2207, 2017 (In Chinese).

33. Hongjiang L, Zhaoliang S, Xitao Y and Dongfu F: Effect of astrocyte activation on the regeneration of optic nerve after injury. Chin J Minimally Invasive Neurosurgery 21: 283-285, 2016 (In Chinese)

34. Hagihara H, Hara M, Tsunekawa K, Nakagawa Y, Sawada M and Nakano K: Tonic-clonic seizures induce division of neuronal progenitor cells with concomitant changes in expression of neurotrophic factors in the brain of pilocarpine-treated mice Brain Res Mol Brain Res 139: 258-266, 2005.
35. Lee TH, Yang JT, Kato H, Wu JH and Chen ST: Expression of brain-derived neurotrophic factor immunoreactivity and mRNA in the hippocampal CA1 and cortical areas after chronic ischemia in rats. J Neurosci Res 76: 705-712, 2004.

36. Lee HJ, Lim IJ, Lee MC and Kim SU: Human neural stem cells genetically modified to overexpress brain-derived neurotrophic factor promote functional recovery and neuroprotection in a mouse stroke model. J Neurosci Res 88: 3282-3294, 2010.

37. Heng W: Expression and significance of brain-derived neurotrophic factor protein and mRNA in rats with intracerebral hemorrhage. Chin J Geriatric Heart Brain Vessel Dis 08: 564-567, 2006 (In Chinese).

38. Ohta K, Ohta M, Mizuta I, Fujinami A, Shimazu S, Sato N, Yoneda F, Hayashi K and Kuno S: The novel catecholaminergic and serotoninergic activity enhancer R-(-)-1-(benzofuran-2-yl)-2-propylaminopentane up-regulates neurotrophic factor synthesis in mouse astrocytes. Neurosci Lett 328: 205-208, 2002.

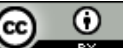

This work is licensed under a Creative Commons Attribution 4.0 International (CC BY 4.0) License. 